Research Article

\title{
Effective Root-Finding Methods for Nonlinear Equations Based on Multiplicative Calculi
}

\author{
Ali Özyapıcı, ${ }^{1}$ Zehra B. Sensoy, $^{1}$ and Tolgay Karanfiller ${ }^{2}$ \\ ${ }^{1}$ Faculty of Engineering, Cyprus International University, Nicosia, Mersin 10, Turkey \\ ${ }^{2}$ Faculty of Education, Cyprus International University, Nicosia, Mersin 10, Turkey \\ Correspondence should be addressed to Ali Özyapıc1; ali.ozyapici@emu.edu.tr
}

Received 29 July 2016; Accepted 14 September 2016

Academic Editor: Liwei Zhang

Copyright (C) 2016 Ali Özyapıc1 et al. This is an open access article distributed under the Creative Commons Attribution License, which permits unrestricted use, distribution, and reproduction in any medium, provided the original work is properly cited.

In recent studies, papers related to the multiplicative based numerical methods demonstrate applicability and efficiency of these methods. Numerical root-finding methods are essential for nonlinear equations and have a wide range of applications in science and engineering. Therefore, the idea of root-finding methods based on multiplicative and Volterra calculi is self-evident. NewtonRaphson, Halley, Broyden, and perturbed root-finding methods are used in numerical analysis for approximating the roots of nonlinear equations. In this paper, Newton-Raphson methods and consequently perturbed root-finding methods are developed in the frameworks of multiplicative and Volterra calculi. The efficiency of these proposed root-finding methods is exposed by examples, and the results are compared with some ordinary methods. One of the striking results of the proposed method is that the rate of convergence for many problems are considerably larger than the original methods.

\section{Introduction}

Ever since Grossman and Katz introduced multiplicative calculus in [1] in the last quarter of nineteenth century, the importance of multiplicative calculi is recently understood by the researchers from the different branches. Especially after the paper by Bashirov et al. [2], some important applications of multiplicative calculus in various applications have been introduced. Some of these are [3] in biomedical image analysis, [4] in complex analysis, [5] in growth phenomena, [6-9] in numerical analysis, [10] in actuarial science, finance, demography, and so forth, [11] in biology, and recently [12] in accounting. It is important to note that first serious application of multiplicative numerical methods was applied by Bilgehan in [13] for representing real time signals in signal processing. Additionally, Volterra (bigeometric by Grossman terminology) calculus also provides a wide range of applications in science. We refer to [14-16] with references therein for further discussions of Volterra (bigeometric) calculus. In this section, we give an overview of some basic concepts of multiplicative and Volterra calculi.

\section{(1) Multiplicative Calculus}

Definition 1. Let $f(x)$ be a function, where $x \in \operatorname{dom}(f)$. If the limit

$$
f^{*}(x)=\lim _{h \rightarrow 0}\left(\frac{f(x+h)}{f(x)}\right)^{1 / h}
$$

exists, then $f$ is called multiplicative differentiable at $x$.

If $f$ is a positive function and the derivative of $f$ at $x$ exists, then $n$th multiplicative derivative of $f$ exists and

$$
f^{*(n)}(x)=\exp \left\{(\ln \circ f)^{(n)}(x)\right\} .
$$

Some properties and basic theorems of multiplicative derivative can be found in $[2,8]$ and papers therein. Referring to Bashirov et al. [2], multiplicative Taylor theorems with one variable can be given, respectively.

Theorem 2 (multiplicative Taylor theorem with one variable). Let $A$ be an open interval and let $f: A \rightarrow \mathbb{R}$ be $n+1$ times 
* differentiable on $A$. Then for any $x, x+h \in A$, there exists a number $\theta \in(0,1)$ such that

$$
\begin{aligned}
f(x+h)= & \prod_{m=0}^{n}\left(f^{*(m)}(x)\right)^{h^{m} / m !} \\
& \cdot\left(f^{*(n+1)}(x+\theta h)\right)^{h^{n+1} /(n+1) !} .
\end{aligned}
$$

Definition 3. Given $x \in \mathbb{R}^{+}=(0, \infty)$, the multiplicative absolute value of $x$ is denoted by $|x|^{*}$ such that

$$
|x|^{*}= \begin{cases}x & \text { if } x \geq 1 \\ \frac{1}{x} & \text { if } x<1\end{cases}
$$

\section{(2) Volterra (Bigeometric) Multiplicative Calculus}

Definition 4. Let $f$ be a positive function over the open interval $(a, b)$. If the limit

$$
f^{\pi}(x)=\frac{d^{\pi} f(x)}{d x}=\lim _{h \rightarrow 0}\left(\frac{f((1+h) x)}{f(x)}\right)^{1 / h}
$$

exists, then $f$ is said to be Volterra type differentiable at $x \in$ $(a, b)$.

In [7], the relation between these two multiplicative calculi can be given as

$$
\begin{aligned}
f^{\pi}(x) & =\left(f^{*}(x)\right)^{x}, \\
f^{\pi \pi}(x) & =\left(f^{* *}(x)\right)^{x^{2}}\left(f^{*}\right)^{x}, \\
f^{\pi(3)}(x) & =\left(f^{*(3)}(x)\right)^{x^{3}}\left(f^{* *}(x)\right)^{3 x^{2}}\left(f^{*}\right)^{x} .
\end{aligned}
$$

Furthermore, necessary concepts on Volterra calculus can easily be derived by using the above relations (6) and (8) together with multiplicative concepts.

Taylor expansion for one variable cannot be obtained easily in Volterra calculus. Few factors of the Volterra type Taylor expansion are deduced, respectively, in [14] and [7] as

$$
\begin{aligned}
& f(x+h) \\
& \quad=f(x) f^{\pi}(x)^{h / x-h^{2} / 2 x^{2}+\cdots} f^{\pi \pi}(x)^{h^{2} / 2 x^{2}-\cdots} \cdots .
\end{aligned}
$$

Recently, the closed form of Volterra Taylor theorem (as Bigeometric Taylor theorem) was presented in the paper [17].

(3) Zero-Finding Methods and Their Applications. The process of determining roots of nonlinear functions is involved in many applications in various fields such as image and audio processing, mathematics, fuzzy systems, and fluid mechanics. In fuzzy systems, it is important to be able to solve nonlinear systems of equations. In fluid mechanics, rootfinding methods arise in finding depth of water. In the case of image processing it is applied to detect the significant local changes (zero crossings) of the intensity levels under the work of edge detection. It is known that accurate, prompt results yield best selection of localization which produce smooth image. In the case of digital audio processing zero crossing points represent the sample at zero amplitude. At any other point, the amplitude of the wave is rising towards its peak or sinking towards zero. It is very important to determine the exact zero crossing in a shortest time interval especially in the case of looping where the joins between the audio must be as smooth as possible.

As mentioned above, the zeros of functions (especially nonlinear functions) are very significant in real applications as well as mathematical applications such as critical points of nonlinear functions. Therefore, using numerical methods are essential in relation to these problems. Newton-Raphson, Chebyshev's, Halley, Broyden, and perturbed methods are some of the important methods for approximating the zeros of functions. The papers [18-20] and many papers therein present recent developments and applications of the ordinary root-finding methods. The main problem of these methods is that the initial quest of each method should be sufficiently close to the root. Moreover, the rate of convergence of these methods is different in many applications.

It is also possible to create alternative methods based on multiplicative calculi which provide larger intervals for initial assumptions with more improved rate of convergence. Firstly, the multiplicative root-finding methods have been discussed in master thesis [21]. For many applications, the use of multiplicative based methods will reduce the amount of computational complexity and the time consumption.

\section{Newton-Raphson Methods via Multiplicative Calculi}

In this section multiplicative root-finding methods based on Newton-Raphson method are alternatively introduced. These methods will provide better performance for many problems.

2.1. Multiplicative Newton-Raphson Method (MN). The starting point of multiplicative Newton formulae will be the corresponding multiplicative Taylor theorems. In order to obtain Volterra taylor theorem, the relationships between multiplicative and Volterra derivatives, stated in Riza et al. [7], were applied.

Theorem 5 (multiplicative Newton-Raphson). Assume that $f \in C^{2}[a, b]$ and there exist a number $m \in[a, b]$ such that $f(m)=1$. If $f^{*}(m) \neq 1$ and $g(x)=x-\ln f(x) / \ln f^{*}(x)$ then there exist a $\delta>0$ such that the sequence $\left\{m_{k}\right\}_{k=1}^{\infty}$ defined by iteration

$$
\begin{aligned}
m_{k}=g\left(m_{k-1}\right)=m_{k-1}-\frac{\ln f\left(m_{k-1}\right)}{\ln f^{*}\left(m_{k-1}\right)} & \\
& \forall k=1,2,3, \ldots
\end{aligned}
$$

will converge to $m$ for any initial value $m_{0} \in[m-\delta, m+\delta]$.

Proof. Since $f(m)=1$, we have $g(m)=m$. To find the point $x$ such that $f(x)=1$ is accomplished by finding a fixed 
point of function $g(x)$. The multiplicative Taylor theorem (Theorem 2) of degree of 1 with its reminder term can be given as

$$
f(x) \approx f\left(m_{0}\right) f^{*}\left(m_{0}\right)^{\left(x-m_{0}\right)} f^{* *}(c)^{\left(x-m_{0}\right)^{2} / 2 !},
$$

where $c \in\left[m_{0}, x\right]$. Substituting $x=m$ into (11) gives

$$
f\left(m_{0}\right) f^{*}\left(m_{0}\right)^{\left(m-m_{0}\right)} f^{* *}(c)^{\left(m-m_{0}\right)^{2} / 2 !} \cong 1
$$

whenever $f(m)=1$. If $m_{0}$ is close enough to $m$, we can consider first two terms of (12) as

$$
f\left(m_{0}\right) f^{*}\left(m_{0}\right)^{\left(m-m_{0}\right)} \approx 1 .
$$

Thus,

$$
m=m_{0}-\left(\frac{\ln f\left(m_{0}\right)}{\ln f^{*}\left(m_{0}\right)}\right) .
$$

Letting $m_{k} \rightarrow m$ and $m_{k-1} \rightarrow m_{0}$ yields (10).

2.2. Volterra (Bigeometric) Newton-Raphson Methods (AVM). The following theorem states the iteration of root-finding algorithms with the convergent criteria in the framework of Volterra calculus.

Theorem 6 (Volterra Newton-Raphson). Assume that $f \in$ $C^{2}[g]$ and there exist a number $v \in[a, b]$ such that $f(v)=1$. If $f^{\pi}(v) \neq 1$ and $g(x)=x-x \ln f(x) / \ln f^{\pi}(x)$, then there exist a $\delta>0$ such that the sequence $\left\{v_{k}\right\}_{k=1}^{\infty}$ defined by iteration

$$
\begin{aligned}
& v_{k}=g\left(v_{k-1}\right)=v_{k-1}-v_{k-1} \frac{\ln f\left(v_{k-1}\right)}{\ln f^{\pi}\left(v_{k-1}\right)} \\
& \qquad \text { for } k=1,2, \ldots
\end{aligned}
$$

will converge to $v$ for any initial value $v_{0} \in[v-\delta, v+\delta]$.

Proof. Insight can be easily given of the proof of multiplicative Newton-Raphson method.

It is very important to note that methods (10) and (15) are identical, because first two terms of multiplicative and Volterra Taylor theorems in the derivation of Newton method yield same iterations.

Alternatively the following theorem can be considered as a modification of Volterra Newton-Raphson method.

Theorem 7 (alternative Volterra Newton-Raphson). Assume that $f \in C^{2}[g]$ and there exist a number $v \in[a, b]$ such that $f(v)=1$. If $f^{\pi}(v) \neq 1, g(x)=x-x \ln f(x) / \ln f^{\pi}(x)$, and $h(x)=(x / 2)\left(3 / 2-\ln f(x) / \ln f^{\pi}(x)+(1 / 2)(1-\right.$ $\left.\left.\ln f(x) / \ln f^{\pi}(x)\right)^{2}\right)$, then there exists $\delta>0$ such that the sequence $\left\{v_{k}\right\}_{k=1}^{\infty}$ defined by iteration

$$
\begin{aligned}
v_{k} & =h\left(v_{k-1}\right)=\frac{v_{k-1}}{2}\left(\frac{3}{2}-\frac{\ln f\left(v_{k-1}\right)}{\ln f^{\pi}\left(v_{k-1}\right)}\right. \\
& \left.+\frac{1}{2}\left(1-\frac{\ln f\left(v_{k-1}\right)}{\ln f^{\pi}\left(v_{k-1}\right)}\right)^{2}\right) \text { for } k=1,2, \ldots
\end{aligned}
$$

will converge to $v$ for any initial value $v_{0} \in[v-\delta, v+\delta]$.
Proof. Insight can be easily given of the proof of multiplicative Newton-Raphson method.

\section{Perturbed Root-Finding Methods via Multiplicative Calculi}

In this section, multiplicative perturbed root-finding methods are derived based on corresponding Taylor theorems. The effort to derive these methods will provide better approximation with less computational time and complexity. The number of perturbed terms in corresponding ordinary perturbed methods (see [20]) should be increased to have the same approximations as multiplicative perturbed methods for many nonlinear equations. The ordinary perturbed method (OP) with two terms can be given as (see $[20,22])$

$$
x_{n+1}=x_{n}-\left[\frac{f\left(x_{n}\right)}{f^{\prime}\left(x_{n}\right)}+\frac{1}{2} \frac{f^{\prime \prime}\left(x_{n}\right)\left(f\left(x_{n}\right)\right)^{2}}{\left(f^{\prime}\left(x_{n}\right)\right)^{3}}\right] \text {. }
$$

The multiplicative perturbed methods with two terms are alternatively derived. Numerical results tabulated in next section demonstrate efficiency of the proposed method for the solutions of nonlinear equations.

3.1. Multiplicative Perturbed Method. We will start with the multiplicative Taylor theorem to derive corresponding multiplicative perturbed method. The first three factors in the multiplicative Taylor theorem can be given as

$$
f(x+h) \approx f(x) f^{*}(x)^{h} f^{* *}(x)^{h^{2} / 2} .
$$

Assume that there exists at least a value $x$ such that the function $f(x)=1$. Representing the root value with perturbation terms as $x=x_{0}+\varepsilon x_{1}+\epsilon^{2} x_{2}$ and substituting them into (18) give

$$
\begin{aligned}
& f\left(x_{0}+\varepsilon x_{1}+\epsilon^{2} x_{2}\right) \approx f\left(x_{0}\right)\left[f^{*}\left(x_{0}\right)\right]^{\varepsilon x_{1}} \\
& \cdot\left(f^{*}\left(x_{0}\right)\right)^{\epsilon^{2} x_{2}}\left(f^{* *}\left(x_{0}\right)\right)^{\varepsilon^{2} x_{1}^{2} / 2}\left(f^{* *}\left(x_{0}\right)\right)^{\varepsilon^{3} x_{1} x_{2}} \\
& \cdot\left(f^{* *}\left(x_{0}\right)\right)^{\varepsilon^{4} x_{2}^{2} / 2} .
\end{aligned}
$$

Omitting the last two terms in (19) and setting 1 give

$$
\begin{aligned}
f\left(x_{0}\right) f^{*}\left(x_{0}\right)^{\varepsilon x_{1}} & =1, \\
\left(f^{*}\left(x_{0}\right)\right)^{\epsilon^{2} x_{2}}\left(f^{* *}\left(x_{0}\right)\right)^{\varepsilon^{2} x_{1}^{2} / 2} & =1 .
\end{aligned}
$$

The solutions of the equations in (20) are, respectively,

$$
\begin{aligned}
\varepsilon x_{1} & =-\frac{\ln f\left(x_{0}\right)}{\ln f^{*}\left(x_{0}\right)}, \\
\epsilon^{2} x_{2} & =\frac{-(1 / 2)\left(\ln f\left(x_{0}\right) / \ln f^{*}\left(x_{0}\right)\right)^{2} \ln f^{* *}\left(x_{0}\right)}{\ln f^{*}\left(x_{0}\right)} .
\end{aligned}
$$


Let the initial estimation of $x$ where $f(x)=1$ to be denoted as $x_{0}$. According to the equations in (21) the second iterative value $x_{1}$ can be computed as

$$
x_{1}=x_{0}-\frac{\ln f\left(x_{0}\right)}{\ln f^{*}\left(x_{0}\right)}-\frac{1}{2} \frac{\ln f^{* *}\left(x_{0}\right)\left(\ln f\left(x_{0}\right)\right)^{2}}{\left(\ln f^{*}\left(x_{0}\right)\right)^{3}} .
$$

Let $x_{1}=x_{n+1}$ and $x_{0}=x_{n}$ in (22). Then,

$$
x_{n+1}=x_{n}-\frac{\ln f\left(x_{n}\right)}{\ln f^{*}\left(x_{n}\right)}-\frac{1}{2} \frac{\ln f^{* *}\left(x_{n}\right)\left(\ln f\left(x_{n}\right)\right)^{2}}{\left(\ln f^{*}\left(x_{n}\right)\right)^{3}} \text {. }
$$

Equation (23) expresses multiplicative perturbed iteration (MP).

3.2. Volterra (Bigeometric) Perturbed Method (VP). We will derive Volterra perturbed method in the framework of Volterra calculus. The starting point is (9) in the form

$$
f(x+h) \approx f(x) f^{\pi}(x)^{h / x} f^{\pi \pi}(x)^{h^{2} / 2 x^{2}} .
$$

Assume that there exists at least a value $x$ such that the function $f(x)=1$. Let the root value with perturbation terms be represented as $x=x_{0}+\varepsilon x_{1}+\epsilon^{2} x_{2}$ and substituting it in (24) gives

$$
\begin{gathered}
f\left(x_{0}+\varepsilon x_{1}+\epsilon^{2} x_{2}\right) \approx f\left(x_{0}\right) f^{\pi}\left(x_{0}\right)^{\varepsilon x_{1} / x_{0}} \\
\cdot f^{\pi}\left(x_{0}\right)^{\epsilon^{2} x_{2} / x_{0}} f^{\pi \pi}\left(x_{0}\right)^{\epsilon^{2} x_{1}^{2} / 2\left(x_{0}\right)^{2}} \\
\cdot f^{\pi \pi}\left(x_{0}\right)^{\varepsilon^{3} x_{1} x_{2} /\left(x_{0}\right)^{2}} f^{\pi \pi}\left(x_{0}\right)^{\varepsilon^{4} x_{2}^{2} / 2\left(x_{0}\right)^{2}} .
\end{gathered}
$$

Omitting the last two terms in (25) and setting it to 1, we have

$$
\begin{aligned}
\varepsilon x_{1} & =x_{0}\left[-\frac{\ln f\left(x_{0}\right)}{\ln f^{\pi}\left(x_{0}\right)}\right], \\
\epsilon^{2} x_{2} & =-x_{0}\left[\frac{\ln f^{\pi \pi}\left(x_{0}\right)\left(\ln f\left(x_{0}\right)\right)^{2}}{2\left(\ln f^{\pi}\left(x_{0}\right)\right)^{3}}\right] .
\end{aligned}
$$

If the initial estimation of $f(x)=1$ is $x_{0}$, the second iterative value $x_{1}$ can be computed by using (26) as

$$
=x_{0}\left[1-\frac{\ln f\left(x_{0}\right)}{\ln f^{\pi}\left(x_{0}\right)}-\frac{1}{2} \frac{\ln f^{\pi \pi}\left(x_{0}\right)\left(\ln f\left(x_{0}\right)\right)^{2}}{\left(\ln f^{\pi}\left(x_{0}\right)\right)^{3}}\right] \text {. }
$$

Let $x_{1}=x_{n+1}$ and $x_{0}=x_{n}$ in (27). Then, we obtain

$$
\begin{aligned}
& x_{n+1} \\
& =x_{n}\left[1-\frac{\ln f\left(x_{n}\right)}{\ln f^{\pi}\left(x_{n}\right)}-\frac{1}{2} \frac{\ln f^{\pi \pi}\left(x_{n}\right)\left(\ln f\left(x_{n}\right)\right)^{2}}{\left(\ln f^{\pi}\left(x_{n}\right)\right)^{3}}\right] .
\end{aligned}
$$

\section{Convergence Criterion of Proposed Methods}

The selection of initial value is also very important for the convergence of the multiplicative root-finding algorithms. In this section, the criterion of the convergence of the proposed methods will be given, which will also lead to a selection of the starting point for any given problem. Before giving the convergence of the multiplicative Newton-Raphson methods due to the initial value, the fixed point theorems should be derived in the multiplicative sense. Additionally, multiplicative Rolle and Mean Value theorems discussed in [2] should be considered. Moreover, Volterra Rolle and Volterra Mean Value theorems, which were firstly given in [16], should explicitly be derived.

Theorem 8. Suppose that $g^{*}$ is defined over $(a, b)$ and let $a$ positive constant $k<e(2.7182818 \ldots)$ with $\left|g^{*}(x)^{g(x)}\right|^{*} \leq k<$ e for all $x \in(a, b)$. Then $g$ has unique fixed point $F$ in $[a, b]$.

Proof. Let $F_{1}$ and $F_{2}$ be two fixed points. Then, by multiplicative Mean Value theorem, there exist $c \in(a, b)$ such that

$$
\left(e^{g(c)}\right)^{*}=\left(\frac{e^{g\left(F_{2}\right)}}{e^{g\left(F_{1}\right)}}\right)^{1 /\left(F_{2}-F_{1}\right)}=e^{\ln \left(e^{F_{2}} / e^{F_{1}}\right)^{1 /\left(F_{2}-F_{1}\right)}}=e,
$$

which contradicts the statement. Therefore, $g(x)$ has a unique fixed point $F$ in $[a, b]$.

By the assumption in multiplicative Newton-Raphson method, $f(m)=1$ and thus $g^{*}(m)=1$. The above theorem produces a sufficient condition for initial value $m_{0}$ to yield a sequence $\left\{m_{k}\right\}$ for $k=0,1,2, \ldots$ for the root of $f(m)=1$, so that $m_{0} \in(m-\delta, m+\delta)$ and $\delta$ can be selected such that

$$
\left(e^{g(x)}\right)^{*}<e
$$

for all $x \in(m-\delta, m+\delta)$.

Hence, the condition in (30) is convergence criteria of the initial value of the proposed multiplicative method.

Analogously, it is possible to derive similar condition for the corresponding Volterra method.

Theorem 9 (Volterra Rolle's theorem). Assume that $f \in$ $C[a, b]$ and $f$ is positive on $[a, b]$. Let $f^{\pi}(x)$ exist for all $x \in$ $(a, b)$. If $f(a)=f(b)=1$, then $\exists c \in(a, b)$ such that

$$
f^{\pi}(c)=1 \text {. }
$$

Proof. It can easily be given by proof of multiplicative Rolle's theorem.

Theorem 10 (Volterra Mean Value theorem). Assume that $f \in C[a, b]$ and $f$ is positive on $[a, b]$. Let $f^{\pi}(x)$ exist for all $x \in(a, b)$. Then, $\exists c \in(a, b)$ such that

$$
f^{\pi}(c)=\left[\frac{f(b)}{f(a)}\right]^{1 /(\ln b-\ln a)} .
$$

Proof. Let us consider the function

$$
F(t)=f(a)\left[\frac{f(b)}{f(a)}\right]^{(\ln t-\ln a) /(\ln b-\ln a)} .
$$


Then $F(a)=f(a)$ and $F(b)=f(b)$. Let $G(t)=F(t) / f(t)$ so that $G(a)=G(b)=1$. By Volterra Rolle's theorem, $\exists c \in$ $(a, b)$ such that

$$
G^{\pi}(c)=\frac{F^{\pi}(t)}{f^{\pi}(t)}=1 .
$$

Hence, $F^{\pi}(t)=f^{\pi}(t)=e^{t[(\ln f(b)-\ln f(a)) / t(\ln b-\ln a)]}$ which gives formula (32).

Theorem 11. Suppose that $g^{\pi}$ is defined over $(a, b)$ and let $a$ positive constant $K \leq e$ with $\left|\left[g(x)^{\pi}\right]\right|^{*} \leq K \leq e^{x}$ for all $x \in$ $(a, b)$, where $e=2.7182818 \ldots$. Then $g$ has unique fixed point $F$ in $[a, b]$.

Proof. It can be easily shown by using Mean Value theorem in Volterra calculus.

Sufficient condition for initial value $v_{0}$ to yield a convergence sequence $\left\{v_{k}\right\}$ for $k=0,1,2, \ldots$ for the root of $f(v)=1$ is that $v_{0} \in(m-\delta, m+\delta)$ and $\delta$ can be selected such that

$$
\left[g(x)^{\pi}\right]<e^{x}
$$

for all $x \in(m-\delta, m+\delta)$.

Hence, condition in (35) is convergence criteria of the initial value of the proposed Volterra method.

\section{Some Numerical Results of Proposed Methods}

In this section, some examples will be considered to reveal the applicability of the introduced methods. Numerical results are reported which indicate that the proposed methods may allow a considerable saving in both the number of step sizes and reduction of computational cost. Besides, the examples consisting of different type of functions reveal the advantages of proposed methods compared to the ordinary methods.

5.1. Comparisons of Multiplicative and Ordinary Methods. It is important to note that finding a zero of the function $h(x)$ at $x=r$ is accomplished by finding the multiplicative root $r$ such that $f(r)=h(r)+1=1$. The numerical results of some nonlinear equations using proposed and ordinary rootfinding methods are listed in Tables 1 and 2.

Displayed in Table 1 is the number of function evaluations required such that $\left|1-f\left(x_{n}\right) / f\left(x_{e}\right)\right|<10^{-15}$. The functions in Table 1 with their roots $x_{e}$, respectively, are

$$
\begin{aligned}
& f_{1}(x)=10 e^{-x^{2}}-1, \quad x_{e}=1.6796306104284499 \\
& f_{2}(x)=\sin x-\frac{1}{2} x, \quad x_{e}=1.8954942670339809 \\
& f_{3}(x)=\tan x-\tanh x
\end{aligned}
$$$$
x_{e}=3.9266023120479185 \text {, }
$$

TABLE 1: OP represents ordinary perturbation iterations, MP represents multiplicative perturbation iterations, and VP represents Volterra perturbation iterations. $x_{0}$ represent initial value. NC states that the given method does not converge for the given zero of function $x_{e}$.

\begin{tabular}{lcccc}
\hline$f\left(x_{n}\right)$ & $x_{0}$ & OP & MP & VP \\
\hline \multirow{2}{*}{$f_{1}$} & 2.5 & NC & 4 & 4 \\
& 0.55 & NC & 6 & 6 \\
\hline \multirow{2}{*}{$f_{2}$} & 0.7 & NC & 5 & NC \\
\hline \multirow{2}{*}{$f_{3}$} & 2 & 3 & 3 & 4 \\
\hline \multirow{2}{*}{$f_{4}$} & 4.5 & 5 & 4 & 3 \\
& 3.5 & 3 & 3 & 3 \\
\hline \multirow{2}{*}{$f_{5}$} & 2.5 & 8 & 4 & 6 \\
& 0.75 & NC & 3 & 4 \\
\hline \multirow{2}{*}{$f_{6}$} & 2.65 & NC & 6 & 6 \\
& 2.25 & 4 & 4 & 4 \\
\hline
\end{tabular}

TABLE 2: NM represents ordinary Newton-Raphson iterations, MN represents multiplicative Newton-Raphson iterations, and AVN represents alternative Volterra Newton-Raphson iterations. $x_{0}$ represent initial value. NC states that the given method does not converge for the given zero of function $x_{e}$.

\begin{tabular}{lcccc}
\hline$f\left(x_{n}\right)$ & $x_{0}$ & NM & MN & AVN \\
\hline \multirow{2}{*}{$f_{1}$} & 2.5 & NC & 5 & 5 \\
& 0.75 & NC & 8 & 11 \\
\hline \multirow{2}{*}{$f_{2}$} & 1.6 & 5 & 5 & 5 \\
& 2 & 4 & 4 & 4 \\
\hline \multirow{2}{*}{$f_{3}$} & 4.5 & 7 & 5 & 5 \\
& 3.5 & 6 & 4 & 5 \\
\hline \multirow{2}{*}{$f_{4}$} & 2.5 & 12 & 4 & 5 \\
& 0.75 & 11 & 4 & 5 \\
\multirow{2}{*}{$f_{5}$} & 3 & 7 & 6 & 6 \\
& 2.25 & 4 & 3 & 4 \\
\hline \multirow{2}{*}{$f_{6}$} & 1.75 & 7 & 6 & 6 \\
& 2.5 & NC & 6 & 5 \\
\hline
\end{tabular}

$$
\begin{array}{r}
f_{4}(x)=x e^{x^{2}}-\sin ^{2} x+3 \cos x-4, \\
x_{e}=1.0651360157761873, \\
f_{5}(x)=e^{-x}+\cos x, \quad x_{e}=1.7461395304080125, \\
f_{6}(x)=x-\ln \left(x^{2}-3\right)-2, \\
x_{e}=1.7461395304080125 .
\end{array}
$$

According to the obtained results, multiplicative rootfinding algorithms can be used effectively and efficiently in real applications mentioned in section one. Moreover, these methods yield better approximations for the nonlinear equations especially when the equations involve exponential, logarithmic, and hyperbolic function. On the other hand, the 
TABLE 3: The number of function evaluations required such that $\left|1-f\left(x_{n}\right) / f\left(x_{e}\right)\right|<10^{-15}$. NC states that the given method does not converge for the given root of (38) $x_{e}=0.5576473009191445$.

\begin{tabular}{cccccccc}
\hline$f\left(x_{n}\right)$ & $x_{0}$ & OP & MP & VP & NM & MN \\
& 1.75 & NC & 4 & 4 & NC & 6 & 6 \\
& 1.25 & NC & 4 & 7 & 14 & 5 & 7 \\
\hline
\end{tabular}

TABLE 4: The number of function evaluations required such that $\left|1-f\left(x_{n}\right) / f\left(x_{e}\right)\right|<10^{-15}$. NC states that the given method does not converge for the given root of (41) $x_{e}=2.1954$.

\begin{tabular}{cccccccc}
\hline$f\left(x_{n}\right)$ & $x_{0}$ & OP & MP & VP & NM & MN & AVN \\
\hline 1.9 & NC & 6 & NC & 7 & 5 & 3 \\
& 1.6 & 3 & 3 & 4 & 3 & 3 \\
\hline
\end{tabular}

ordinary methods can give more accurate results especially for polynomial equations. Thus, the method should be selected according to the functions appeared in the equations.

5.2. Realistic Applications. In this subsection, two examples will be considered to demonstrate possible impacts of the introduced methods to science and engineering.

Example 12. The process in which chemicals interact to form new chemicals with different compositions is called chemical reactions. This process is the results of chemical properties of the element or compound causing changes in composition. These chemical changes are chemists' main purpose. It is important to mention a remark from [23] where "the two questions that must be answered for a chemically reacting system are: (1) what changes are expected to occur and (2) how fast will they occur?" This is an indication that mathematical representations are very important for chemical investigations. Consequently, a chemical reaction is mainly represented by a chemical equation, which represents the change from reactants to products. This process, generally, involves nonlinear functions, so, it is compulsory to use and apply numerical approaches. Exemplarily, suppose that a chemical reaction is made and the concentration of a particular ion at the time $t$ is given by a nonlinear function:

$$
f(x)=5 e^{-3 x}+e^{-5 x}
$$

If we are interested in when this concentration will be onehalf of its value at initial time 0 , we need to solve this problem numerically. If $f(0)=2$ as an initial assumption, this will be equivalent to finding a root of nonlinear equation:

$$
5 e^{-3 x}+e^{-5 x}-1=0 .
$$

Usually, chemists tend to use ordinary numerical methods to estimate the root of (38). However, the multiplicative base methods whose effectiveness was proved in problems that involve exponential functions should not be disregarded in many applications. The superiority of the multiplicative numerical methods can be easily observed for this problem by Table 3 .
Example 13. The Rayleigh function, which corresponds to Rayleigh distribution,

$$
f(x)=\frac{x}{\sigma} e^{-x^{2} / 2 \sigma^{2}}, \quad \text { for arbitrary } a, b, c \in R
$$

plays an important role in magnetic resonance imaging (MRI) and probability theory. It is a striking example in order to show the efficiency of the multiplicative and Volterra rootfinding methods. Assume that the density function of the continuous random variable $X$ on the interval $[0, \infty)$ is given by

$$
f(x)=x e^{-x^{2} / 2}, \quad \text { where } \sigma=1 .
$$

It may be interesting to consider the root of the equation

$$
\alpha-f(x)=\alpha-x e^{-x^{2} / 2}=0, \quad 0 \leq \alpha \leq 1
$$

which estimates the point $x$ according to the given probability $\alpha$ of an event in $X$. We attempt to use root-finding algorithms for (41) for $\alpha=0.19720178928946303$ which will be given by Table 4.

\section{Conclusion}

In this study, the multiplicative and Volterra based rootfinding methods are presented. These methods were tested for some nontrivial problems and compared with the original root-finding method. The results show that in certain problems the multiplicative and/or Volterra methods give more accurate results compared to the original root-finding methods. Especially, the examples showed that the nature of the underlying calculus plays an important role in approximating the zeros of the function.

The selection of the initial value is very important for the convergence of the iteration. Two theorems in Section 4 indicate the conditions for convergence related to multiplicative and Volterra methods, respectively. Section 4 also highlights optimal selection of the initial value. Evidently in certain situations, the multiplicative and/or Volterra methods converge faster compared to the ordinary methods. Particularly, it can be easily observed that multiplicative and Volterra NewtonRaphson methods are more accurate than ordinary NewtonRaphson method in many applications. Therefore, these 
methods based on multiplicative calculi have proven their importance in the process of numerical approximations of nonlinear equations. Also, the numerical results obtained in the paper encourage the usage of multiplicative and Volterra methods for solving nonlinear equations.

\section{Competing Interests}

The authors declare that there is no conflict of interests regarding the publication of this paper.

\section{References}

[1] M. Grossman and R. Katz, Non-Newtonian Calculus, Lee Press, Pigeon Cove, Mass, USA, 1972.

[2] A. E. Bashirov, E. M. Kurpınar, and A. Özyapıc1, "Multiplicative calculus and its applications," Journal of Mathematical Analysis and Applications, vol. 337, no. 1, pp. 36-48, 2008.

[3] L. Florack and H. V. Assen, "Multiplicative calculus in biomedical image analysis," Journal of Mathematical Imaging and Vision, vol. 42, no. 1, pp. 64-75, 2012.

[4] A. Uzer, "Multiplicative type complex calculus as an alternative to the classical calculus," Computers \& Mathematics with Applications, vol. 60, no. 10, pp. 2725-2737, 2010.

[5] D. A. Filip and C. Piatecki, "A non-Newtonian examination of the theory of exogenous economic growth," CNCSISUEFISCSU (project number PNII IDEI 2366/2008) and Laboratoire d.Economie d.Orleans (LEO), 2010.

[6] E. Misirli and Y. Gurefe, "Multiplicative adams bashforthmoulton methods," Numerical Algorithms, vol. 57, no. 4, pp. 425-439, 2011.

[7] M. Riza, A. Özyapıc1, and E. Misirli, "Multiplicative finite difference methods," Quarterly of Applied Mathematics, vol. 67, no. 4, pp. 745-754, 2009.

[8] A. Ozyapici and E. Misirli, "Exponential approximations on multiplicative calculus," Proceedings of the Jangjeon Mathematical Society, vol. 12, no. 2, pp. 227-236, 2009.

[9] A. Ozyapici and B. Bilgehan, "Finite product representation via multiplicative calculus and its applications to exponential signal processing," Numerical Algorithms, vol. 71, no. 2, pp. 475-489, 2016.

[10] A. E. Bashirov, E. Misırlı, Y. Tandoğdu, and A. Özyapıcı, "On modeling with multiplicative differential equations," Applied Mathematics, vol. 26, no. 4, pp. 425-438, 2011.

[11] J. Englehardt, J. Swartout, and C. Loewenstine, "A new theoretical discrete growth distribution with verification for microbial counts in water," Risk Analysis, vol. 29, no. 6, pp. 841-856, 2009.

[12] H. Özyapıc1, İ. Dalc1, and A. Özyapıcı, "Integrating accounting and multiplicative calculus: an effective estimation of learning curve," Computational and Mathematical Organization Theory, 2016.

[13] B. Bilgehan, "Efficient approximation for linear and non-linear signal representation," IET Signal Processing, vol. 9, no. 3, pp. 260-266, 2015.

[14] D. Aniszewska, "Multiplicative Runge-Kutta methods," Nonlinear Dynamics, vol. 50, no. 1-2, pp. 265-272, 2007.

[15] W. Kasprzak, B. Lysik, and M. Rybaczuk, Dimensions, Invariants Models and Fractals, Ukrainian Society on Fracture Mechanics, SPOLOM, Wroclaw-Lviv, Poland, 2004.
[16] M. Grossman, Bigeometric Calculus: A System with a ScaleFree Derivative, Archimedes Foundation, Rockport, Mass, USA, 1983.

[17] M. Riza and B. Eminaga1, "Bigeometric calculus—a modelling tool," https://arxiv.org/abs/1402.2877vl.

[18] M. A. Noor, W. A. Khan, and A. Hussain, "A new modified Halley method without second derivatives for nonlinear equation," Applied Mathematics and Computation, vol. 189, no. 2, pp. 12681273, 2007.

[19] A. Ramli, M. L. Abdullah, and M. Mamat, "Broyden's method for solving fuzzy nonlinear equations," Advances in Fuzzy Systems, vol. 2010, Article ID 763270, 6 pages, 2010.

[20] M. Pakdemirli, H. Boyac1, and H. A. Yurtsever, "Perturbative derivation and comparisons of root-finding algorithms with fourth order derivatives," Mathematical and Computational Applications, vol. 12, no. 2, pp. 117-124, 2007.

[21] E. Misirli and Y. Gurefe, Multiplicative calculus and its applications [M.S. thesis], Turkish Council of Higher Education, 2009, Thesis No: 252639.

[22] M. Pakdemirli and H. Boyac1, "Generation of root finding algorithms via perturbation theory and some formulas," Applied Mathematics and Computation, vol. 184, no. 2, pp. 783-788, 2007.

[23] M. E. Davis and R. J. Davis, Fundamentals of Chemical Reaction Engineering, MGraw-Hill Companies, 2003. 


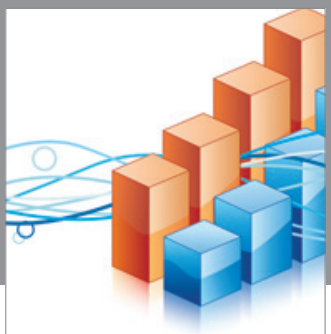

Advances in

Operations Research

vatem alat4

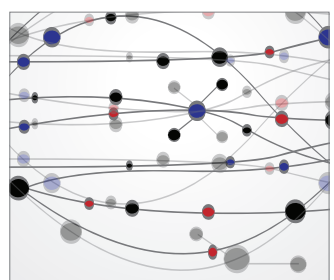

\section{The Scientific} World Journal
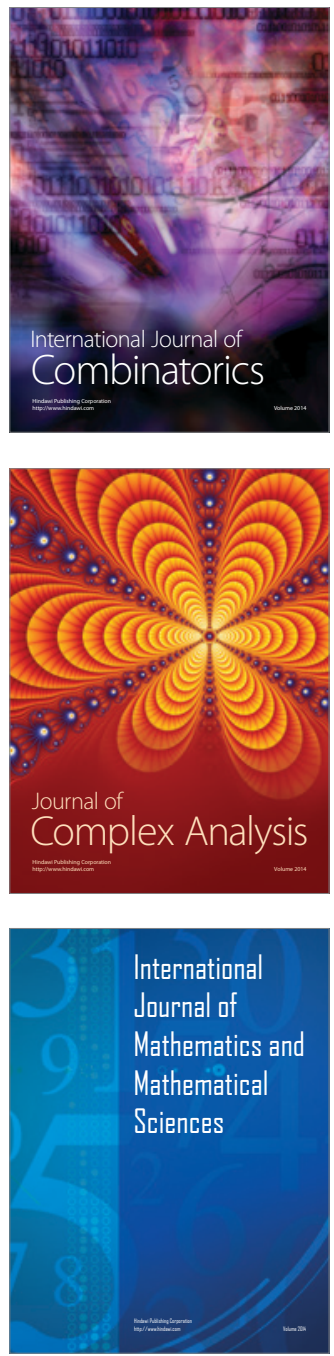
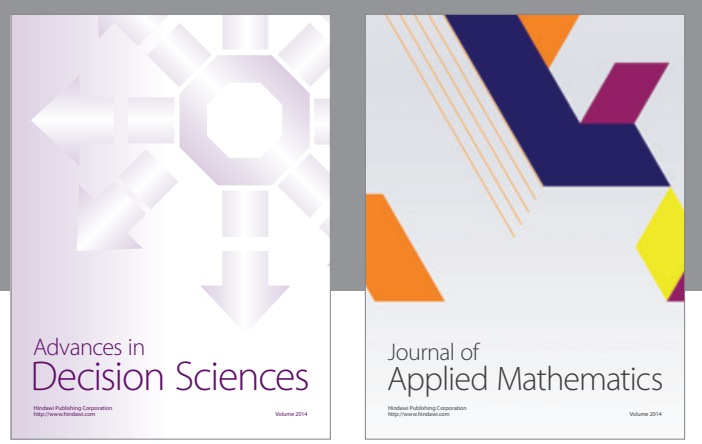

Algebra

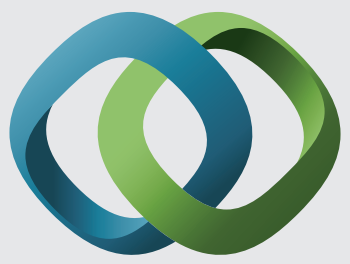

\section{Hindawi}

Submit your manuscripts at

http://www.hindawi.com
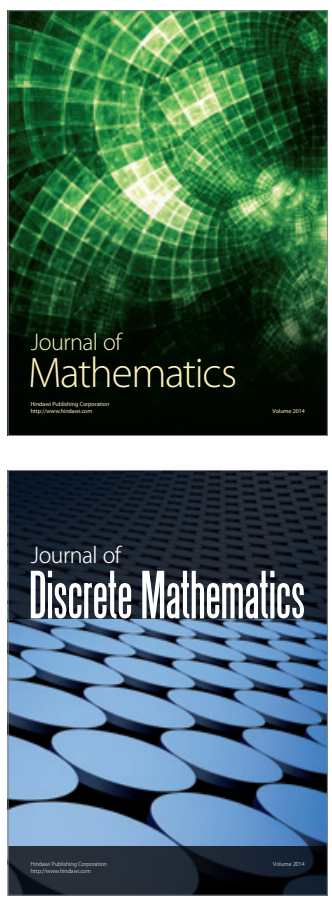

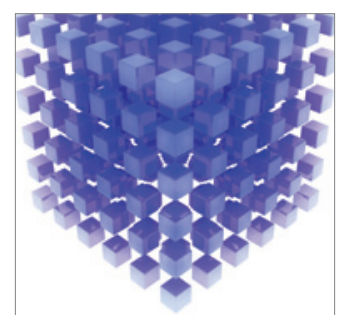

Mathematical Problems in Engineering
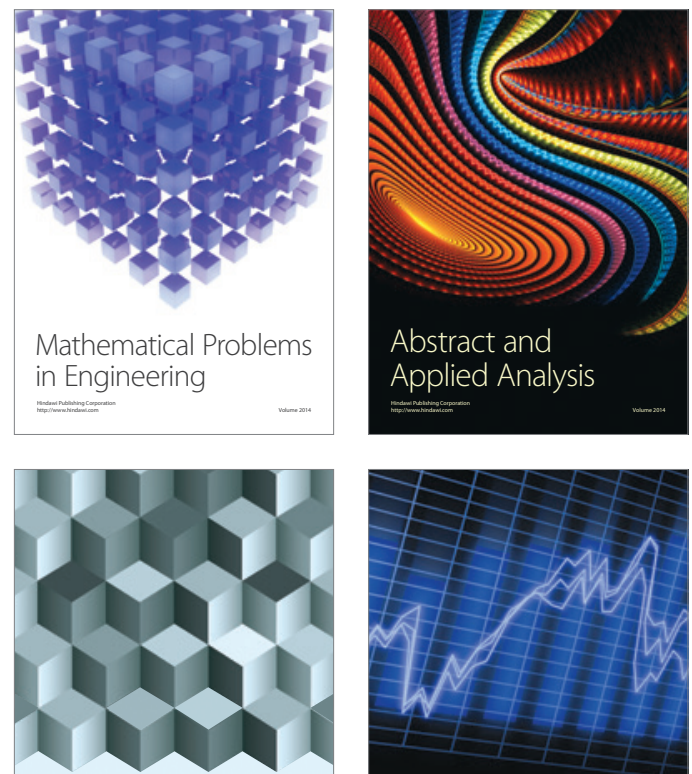

Journal of

Function Spaces

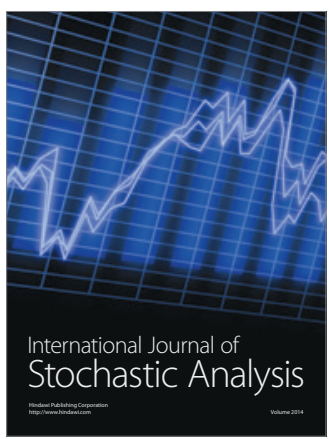

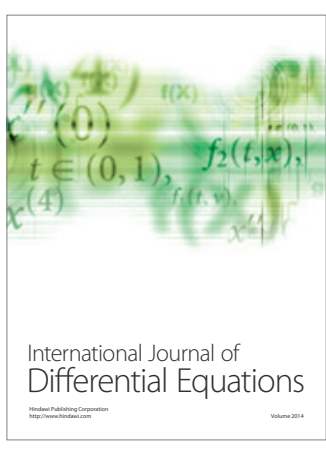
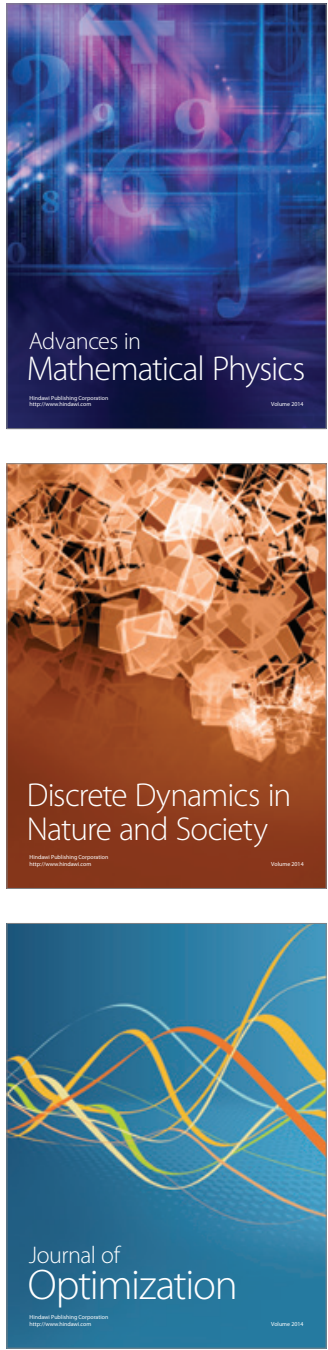